\title{
Practical aspects of implementing three-dimensional tomography inversion for volumetric flame imaging
}

\author{
Weiwei Cai, ${ }^{1}$ Xuesong $\mathrm{Li}^{2}{ }^{2}$ and $\mathrm{Lin} \mathrm{Ma}^{3, *}$ \\ ${ }^{1}$ Virginia Tech, Blacksburg, Virginia 24060, USA \\ ${ }^{2}$ Department of Aerospace and Ocean Engineering, Virginia Tech, Blacksburg, Virginia 24061, USA \\ ${ }^{3}$ Laser Diagnostic Lab, Department of Aerospace and Ocean Engineering, Virginia Tech, Blacksburg, \\ Virginia 24061, USA \\ ${ }^{*}$ Corresponding author: LinMa@vt.edu
}

Received 19 September 2013; accepted 20 October 2013;

posted 23 October 2013 (Doc. ID 197784); published 18 November 2013

\begin{abstract}
Instantaneous three-dimensional (3D) measurements have been long desired to resolve the spatial structures of turbulent flows and flame. Previous efforts have demonstrated tomography as a promising technique to enable such measurements. To facilitate the practical application, this work investigated four practical aspects for implementing 3D tomographic under the context of volumetric combustion diagnostics. Both numerical simulations and controlled experiments were performed to study: (1) the termination criteria of the inversion algorithm; (2) the effects of regularization and the determination of the optimal regularization factor; (3) the effects of a number of views; and (4) the impact of the resolution of the projection measurements. The results obtained have illustrated the effects of these practical aspects on the accuracy and spatial resolution of volumetric tomography. Furthermore, all these aspects are related to the complexity and implementing cost (both hardware cost and computational cost). Therefore, the results obtained in this work are expected to be valuable for the design and implementation of practical 3D diagnostics. (C) 2013 Optical Society of America

OCIS codes: $\quad$ (280.1740) Combustion diagnostics; (100.6890) Three-dimensional image processing; (120.0280) Remote sensing and sensors.

http://dx.doi.org/10.1364/AO.52.008106
\end{abstract}

\section{Introduction}

Three-dimensional (3D) measurements have been long desired for the study of many thermal-fluid topics to resolve the 3D spatial structures inherent in turbulent flows [1,2]. Furthermore, instantaneous measurements with proper temporal resolution are of particular importance due to the dynamic and transient nature of turbulent flows [1,2]. Possible options that can potentially meet both the spatial and temporal requirements seem to be very limited

1559-128X/13/338106-11\$15.00/0

(C) 2013 Optical Society of America and most of the existing efforts can be broadly divided into two categories $[\underline{3}, \underline{4}]$. The first category of techniques obtains 3D measurements by rapidly scanning a planar imaging technique such as Mie scattering [5] or laser induced incandescence [6]. The second category of techniques obtains $\overline{3 D}$ measurements volumetrically by performing a 3D tomography [7-10]. A comprehensive survey and comparison of these possible options is beyond the scope of this current paper, and an independent review of this topic would be of significant importance and value for optical diagnosticians. This current work therefore directly focuses on the tomography approach. More specifically, the current work 
examines several practical aspects of implementing 3D tomographic inversions under the context of combustion measurements based on previous results. Existing tomography inversion methods are predominately developed by and for the medical imaging community [11]. Consequently, direct application of these methods to flow and combustion imaging often results in nonoptimal performance due to both the fundamental and practical differences between flow/flame and medical imaging. This work particularly discusses the following four practical aspects of $3 \mathrm{D}$ tomographic inversion under the context of volumetric combustion measurements.

First, this paper discusses the termination criteria of inversion methods and their effects on the accuracy and efficiency of tomographic reconstruction. An effective termination criterion is an important aspect of any inversion method, because it directly affects the computational cost and the reconstruction accuracy. An ideal criterion should be one that guarantees convergence and terminates immediately when further calculation provides negligible improvements. Theories are available to analyze the termination criteria, but practical issues such as measurement noise and inconsistent projection data restrict the usefulness of the theories and the termination criteria often need to be determined empirically $[9,11,12]$. This work studied the termination criteria using both simulated and experimental data, and demonstrated the effective termination of an inversion method based on the simulated annealing (SA) algorithm.

Second, this paper discusses the effects of regularization on the reconstruction of various flames. Regularization, the incorporation of available $a$ priori information in the inversion, is an effective technique to improve the performance of tomography, applicable for both medical [13] and combustion imaging [14,15]. However, after studying various regularization techniques on various flame patterns, the results obtained in this work show that the regularization techniques developed for medical imaging do not apply effectively for flame measurements due to the structural differences between medical targets and flames. For example, the former typically features sharp edges while the latter (especially a highly turbulent flame) features distributed and irregular patterns.

Third, this paper discusses the effects of number of views on reconstruction quality. In contrast to medical imaging where projection data at many views (e.g., more than thousands) are available as input for the inversion, projection data in combustion applications are often available at a very limited number of views, both because of the dynamic nature of flames and the practical difficulty of obtaining optical access [16-18]. Past works have performed both computational and theoretical studies on the effects of the number of views on the spatial resolution and fidelity of the reconstruction $[9,19]$. They also explored possible ways of obtaining more views in combustion systems using imaging fibers [20]. This work examined the effects of a number of views for tomographic 3D combustion imaging combining computational, theoretical, and experimental results.

Fourth, this paper discusses the effects of the resolution of the projections measurements on the spatial resolution and fidelity of the reconstruction. Projection data for 3D tomography are recorded (by CCD cameras for example) with finite spatial resolution. The projection measurements are often binned to enhance the signal-to-noise ratio in combustion applications, and such binning degrades the spatial resolution of the projection data. This work therefore, examined the effects of CCD resolution and binning on 3D tomographic inversion.

The rest of the paper is organized as follows. Section 2 summarizes the mathematical formulation and experimental arrangement used in this work. Section 3 reports the results obtained in all four aspects from both numerical simulation and experimental studies. Section 4 summarizes the paper and discusses our ongoing research.

\section{Mathematical Formulation and Experimental Arrangement}

\section{A. Problem Formulation and Inversion Algorithms}

The mathematical formulation of 3D tomography has been previously detailed elsewhere [10,21], and a brief summary is provided here to facilitate the discussion. Figure 1 schematically illustrates the mathematical formulation. As shown, the volumetric distribution of the target quantity [denoted as $F(x, y, z)$ ] is discretized into voxels. For example, in this work, the experimental work relied on the chemiluminescence emission of $\mathrm{CH}^{*}$ and $F(x, y, z)$, hence represents the concentration of $\mathrm{CH}^{*}$ in this case. But as to be seen, the mathematical formulation is general and applicable to tomography based on other signal generation processes as well, including chemiluminescence from other flame radicals (or emission spectroscopy in general), Mie scattering, or laser-induced fluorescence (LIF). Thus

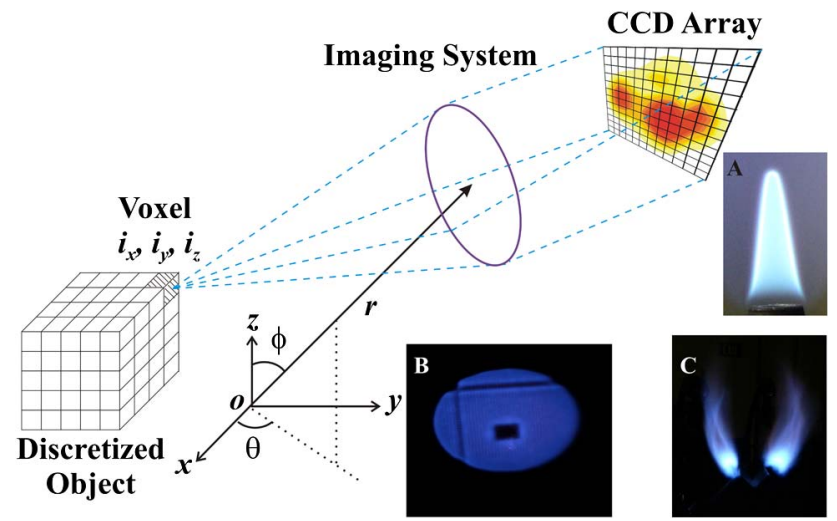

Fig. 1. Mathematical formulation of the TC problem and example flames used to validate the TC technique experimentally. 
we expect the results (at least the simulation results) to be applicable to tomographic imaging based on other signal generation processes. An image system collects the signal to be recorded by a CCD array, and the two-dimensional image formed on the CCD array is called a projection $(P)$. The projection formed on the CCD is given by

$$
\begin{aligned}
P(r, \theta, \phi)= & \sum_{i_{x}}^{N_{x}} \sum_{i_{y}}^{N_{y}} \sum_{i_{z}}^{N_{z}} F\left(x_{i}, y_{i}, z_{i}\right) \\
& \cdot \operatorname{PSF}\left(x_{i}, y_{i}, z_{i} ; r, \theta, \phi\right),
\end{aligned}
$$

where $r$ (distance to the origin), $\theta$ (azimuth angle), and $\phi$ (inclination angle) specify the orientation and location of the projection measurement as shown in Panel a; $i_{x}, i_{y}, i_{z}$ are the indices of the voxel centered at $\left(x_{i}, y_{i}, z_{i}\right) ; N_{x}, N_{y}$, and $N_{z}$ are the total number of grids along the $x, y, z$ direction, respectively; and PSF represents the point spread function defined as the projection formed by a point source located at $\left(x_{i}, y_{i}, z_{i}\right)$ with unity intensity. Equation (1) essentially calculates the projection as a weighted summation of the PSF across all voxels with the sought function as the weight. The 3D tomography problem can now be formulated as:

Given a set of projections $(P \mathrm{~s})$ measured at various $r, \theta$, and $\phi$, find $F(x, y, z)$.

As mentioned above, this formulation is generally applicable to signals generated from various mechanisms including chemiluminescence [10], emission [18], scattering [22,23], and LIF [24]. In the case where the signal is angle dependent (like Mie scattering), the PSF can be modified to include the scattering phase function and then Eq. (1) remains valid. However, note that Eq. (1) is limited to linear signals, and is not applicable to problems with nonlinear effects such as multiple scattering.

Various algorithms have been developed to solve the tomographic inversion problem as formulated above [11], and our work has implemented several of these algorithms including the algebraic reconstruction technique (ART) as described in [9], the multiplicative algebraic reconstruction technique (MART) as described in [25], and the ordered subset expectation maximization (OSEM) algorithm as described in [26]. We have also developed a new algorithm based on the SA technique [27] to solve the 3D tomography problem, and our new algorithm tomographic inversion by SA, is code named TISA. The TISA algorithm formulates the tomography problem into an optimization problem as shown below:

$$
\begin{aligned}
& \text { minimize } D=\sum_{r, \theta, \phi}\left[P_{m}(r, \theta, \phi)-P_{c}(r, \theta, \phi)\right]^{2} \\
& \text { with respect to } F(x, y, z),
\end{aligned}
$$

where $P_{m}$ represents the measured projections at $(r, \theta, \phi), P_{c}$ the projection calculated at $(r, \theta, \phi)$ with a given distribution according to Eq. (1), and $D$ therefore, the overall difference between the measured and calculated projection. Equation (2) is then minimized by the SA technique. Before comparing the various algorithms on the four specific aspects aforementioned, we make some general comments based on our previous results. Combustion imaging presents a set of unique challenges and also opportunities for the development of inversion algorithms. We have performed numerical comparisons of these algorithms on a range of phantoms (i.e., targets $F_{\mathrm{s}}$ that are artificially created) and different signal generating mechanisms, and the results have suggested reasonable performance from all algorithms and also limitations on phantoms featuring highly turbulent and irregular patterns $[10,12,14,23,28,29]$. The first challenge of combustion imaging involves the availability of projection data at a very limited number of views, ranging from $2[15,16]$ to about 50 $[9,18,20,30,31]$. In contrast, other applications (e.g., medical imaging) have significantly more projections (thousands and more) available. Therefore, the desired algorithm should converge under limited projection data (which are often also noisy). The second challenge involves the computational cost needed for processing and analyzing flow and combustion measurements. Tomographic inversion itself is computationally intensive, comparable to computational fluid dynamics (CFD) in many ways [32]. The computational cost is further compounded by the need to take measurements at high speed (multikilohertz) to resolve the temporal dynamics of turbulent flows and flames. Therefore, the desired algorithm should be computationally efficient and also amiable for large scale parallelization [32-34]. Despite these challenges, flows and flames also present some unique opportunities for tomographic imaging, and the ideal algorithm should be able to incorporate these opportunities. For example, unlike medical targets, flows and flames follow a set of governing equations, presenting an opportunity to incorporate these governing equations in the inversion process to improve the reconstruction accuracy $[\underline{32}, \underline{35]}$ and reduce the computational cost [36].

\section{B. Experimental Arrangement}

The experimental study in this work was performed using laboratory flames and multiview arrangement to conduct $3 \mathrm{D}$ tomographic chemiluminescence (TC), similar to that discussed in [10]. Chemiluminescence emitted from $\mathrm{CH}^{*}$ radicals in the flame from various view angles was recorded either sequentially by one camera (if the flame is stable) or simultaneously by multiple cameras (if the flame is unstable). Then based on the measured projections, a tomographic reconstruction was performed to obtain the 3D flame structure.

Three of the example flames used in the experimental study are shown in Fig. 1: (A) a simple laminar flame generated by a Bunsen burner, (B) a patterned flame generated by a customized 
McKenna burner, and (C) a v-gutter stabilized flame. These flames were either designed to have stable and well-defined spatial features (such as flames A and B) to quantitatively validate $3 \mathrm{D}$ measurements base on TC, or designed to be turbulent and dynamics (such as flame C) to demonstrate TC's temporal resolution. For example, flame A had a known cone shape and a flame front thickness of $0.30 \mathrm{~mm}$. Flame $\mathrm{B}$ is a stable and disk-like flame with a diameter of $\sim 61 \mathrm{~mm}$ and a thickness of $\sim 1 \mathrm{~mm}$. A honeycomb was place on the burner to create controlled patterns on the flames. The honeycomb's cells are squares with size of $1.25 \times 1.25 \mathrm{~mm}$ and certain cells were blocked to create the desired pattern. Various patterns have been created and studied in this work. Flame B shown in Fig. 1 has patterns of a rectangular region with a size of $8.75 \times 10 \mathrm{~mm}$, a column of cells to form a vertical line with $1.25 \mathrm{~mm}$ thickness, and two rows of cells to form a horizontal line with and $2.5 \mathrm{~mm}$ thickness. After the tomographic reconstruction, these spatial features (such as the location, thickness, and size of the flame front) were extracted and compared to the known values to quantify the accuracy of the TC measurements, as will be detailed in Section 3 .

In summary, this section introduces the mathematical formulation and experimental approach used in this work. Based on these, the next section will examine the four specific aspects of $3 \mathrm{D}$ flame imaging quantitatively.

\section{Results and Discussion}

Both numerical and experimental studies have been performed for the discussions in this section. The numerical study used various phantoms, with four of them shown in Fig. 2, and the experimental study was performed as discussed in Section 2.B. The phantoms were designed with the intention to simulate the phantoms used in this work, so that
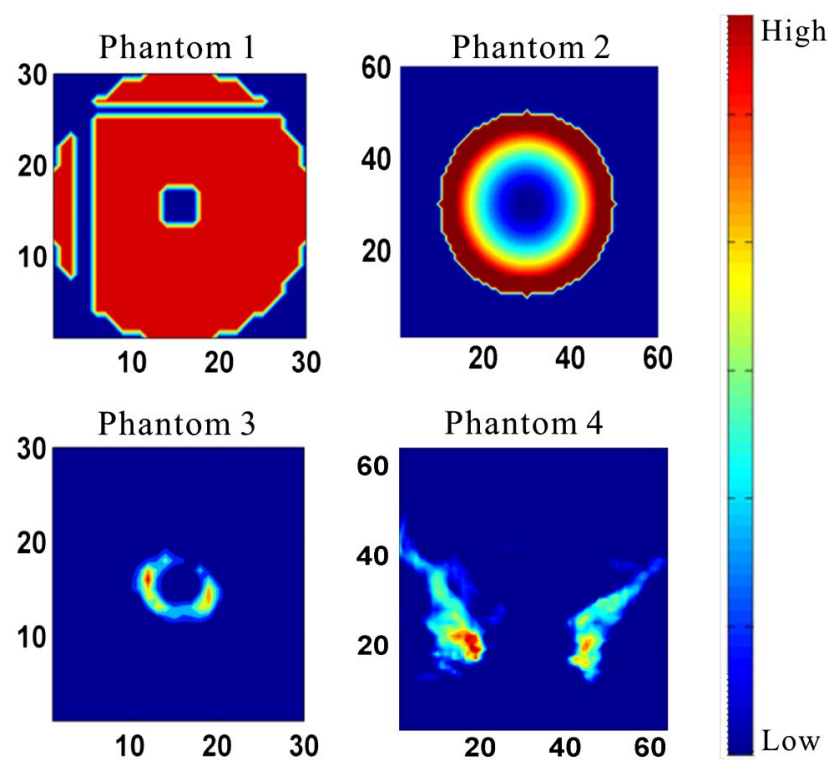

Fig. 2. Phantoms used for numerical simulations. the performance of the tomography measurements can be evaluated both experimentally and numerically. For example, phantom 1 in Fig. 2 was created to simulate the patterned McKenna burner flame generated experimentally, phantom 2 to simulate the stable Bunsen flame, phantom 3 to simulate a turbulent jet flame, and phantom 4 to simulate a turbulent flame stabilized by a v-gutter. Though Fig. 2 shows one layer of each phantom, all phantoms were created and used volumetrically in this work. Also note that the same color scale as used here in Fig. 2 (red and blue, respectively, correspond to high and low $\mathrm{CH}^{*}$ concentration) is applied throughout this work.

Before discussing any of the specific aspects, we first establish that the inversion algorithms are insensitive to the initial starting condition. Various initial guesses were used to start the algorithms tested, including TISA, ART, MART, and OSEM. All these algorithms were observed to be insensitive to the initial guess. Figure 3 shows a set of example results where the TISA algorithm was applied to phantom 4 with three different initial guesses of the target $F$ : a 3D Gaussian distribution, a 3D uniform distribution, and a 3D random distribution. As Fig. 3 shows, the function value (normalized by the largest $F$ observed) changed differently during iterations with different initial guesses, but the reconstruction error $(e)$ all converged to the same value. This work defines the following reconstruction error to quantify the inversion accuracy:

$$
e=\frac{\sum_{i_{x}} \sum_{i_{y}} \sum_{i_{z}}\left|F_{i_{x}, i_{y}, i_{z}}^{\mathrm{rec}}-F_{i_{x}, i_{y}, i_{z}}\right|}{\sum_{i_{x}} \sum_{i_{y}} \sum_{i_{z}}\left|F_{i_{x}, i_{y}, i_{z}}\right|},
$$

where $F^{\text {rec }}$ represents the reconstructed distribution of the target radical. Similar behavior was observed with all algorithms on both phantoms and experimental measurements.

\section{A. Termination Criterion}

An effective termination criterion is important for any inversion method because it directly affects the computational cost and also the reconstruction

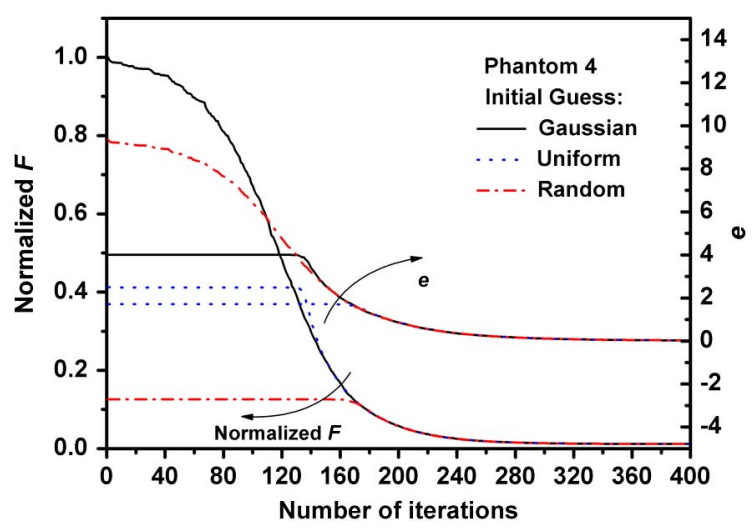

Fig. 3. Insensitivity to initial guess. 
accuracy. An ideal termination criterion is one that guarantees convergence while aborting immediately when further calculation provides negligible improvements. Unfortunately, there is no universal criterion, to our knowledge, that works effectively for all practical applications where empirical criteria are often used. As an example, the following termination criterion has been used for the ART algorithms [37]:

$$
\begin{aligned}
& \left|\sum_{i_{x}}^{N_{x}} \sum_{i_{y}}^{N_{y}} \sum_{i_{z}}^{N_{z}} F^{k}\left(x_{i}, y_{i}, z_{i}\right)-\sum_{i_{x}}^{N_{x}} \sum_{i_{y}}^{N_{y}} \sum_{i_{z}}^{N_{z}} F^{k-1}\left(x_{i}, y_{i}, z_{i}\right)\right| \\
& \quad \leq \varepsilon \cdot \beta \cdot \sum_{i_{x}}^{N_{x}} \sum_{i_{y}}^{N_{y}} \sum_{i_{z}}^{N_{z}} F^{k}\left(x_{i}, y_{i}, z_{i}\right),
\end{aligned}
$$

where $F^{k}$ and $F^{k-1}$ are the reconstructed $F$ in the $k$ th and $(k-1)$ th iteration, respectively; $\varepsilon$ is a small positive number and was empirically suggested to be in the range of $\left[10^{-6}, 10^{-3}\right]$; and $\beta$ is the relaxation factor in the ART algorithm. This criterion essentially terminates the ART algorithm when the overall change in $F$ during two consecutive iterations is below a small proportion of the overall magnitude of the reconstructed $F$. Figure 4 illustrates the limitation of this criterion by simultaneously tracking $D$ and $e$ during ART iterations. Here $D$ refers to the overall difference between the measured and calculated projections during the $k$ th iteration, i.e.,

$$
D=\sum_{r, \theta, \phi}\left|P_{m}^{k}(r, \theta, \phi)-P_{c}^{k}(r, \theta, \phi)\right|^{2},
$$

where $P_{m}^{k}$ and $P_{c}^{k} P_{m}$ stand for measured and calculated projection obtained by the end of the $k$ th ART iteration. As Fig. 4 shows, $D$ decreases monotonically with $k$, which increases monotonically with decreasing $\varepsilon$ because more iterations are needed to find $F$ that can better match the calculated projections to the measured ones. However, $e$ does not decrease monotonically with increasing $k$ (and consequently not decreasing $\varepsilon$ either). In the results shown in Fig. 4 , setting $\varepsilon=10^{-3}$ terminated the inversion

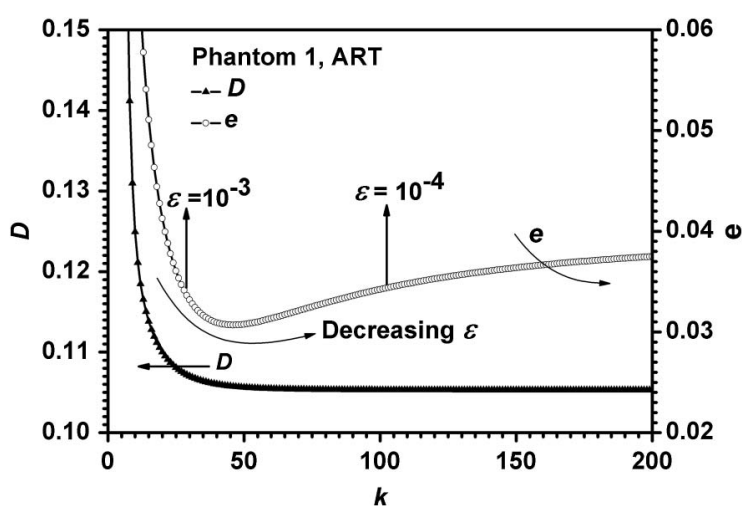

Fig. 4. Evolution of $e$ and normalized residual illustrating issues with termination criterion in the ART algorithm. too early, before the minimal $e$ was reached. In contrast, setting $\varepsilon=10^{-4}$ terminated the inversion too late, after it passed the minimal $e$ and resulted in a less accurate reconstruction after almost $4 \times$ more computational cost compared to the criterion with $\varepsilon=10^{-3}$. The results obtained in Fig. $\underline{4}$ were phantom 1 , eight randomly positioned views and 5\% artificial Gaussian noise in projections. The ART, MART, and OSEM algorithms were tested on various phantoms with different termination criteria. The results confirmed the difficulty of designing an effective termination criterion. All the algorithms showed success on some cases, and also encountered issues like those illustrated in Fig. $\underline{4}$ on other cases.

In comparison, the new TISA algorithms that we developed can be terminated consistently with a simple criterion in all the cases we tested. The TISA algorithm solves Eq. (2) by the SA algorithm [38]. The SA algorithm is a proven technique for minimizing complicated functions. The SA algorithm seeks the minimum of a function by simulating the way liquids anneal and crystallize, a natural process during which a large number of molecules find the state of minimal total energy [27]. The SA algorithm minimizes $D$, defined in Eq. (2) also by iterations, and the following criterion was found to be effective:

$$
\left|D^{k}-D^{k-1}\right| \leq \varepsilon \cdot D^{k}
$$

where $D^{k}$ and $D^{k-1}$ are the difference between the simulated and measured projections as defined in Eq. (2) during the $k$ th and $k-1$ th iteration, respectively. Similar to Fig. 4 , Fig. 5 tracks $D$ (normalized by $D^{1}$ ) and $e$ simultaneously under the same conditions as those used in Fig. 4. The results in Fig. 5 show that, with Eq. (6), both $\bar{D}$ and $e$ decreased monotonically with decreasing $\varepsilon$ (i.e., increasing $k$ and computation cost) in the TISA algorithm. Results obtained in other cases with the TISA algorithm showed the same trend as seen in Fig. $\underline{5}$.

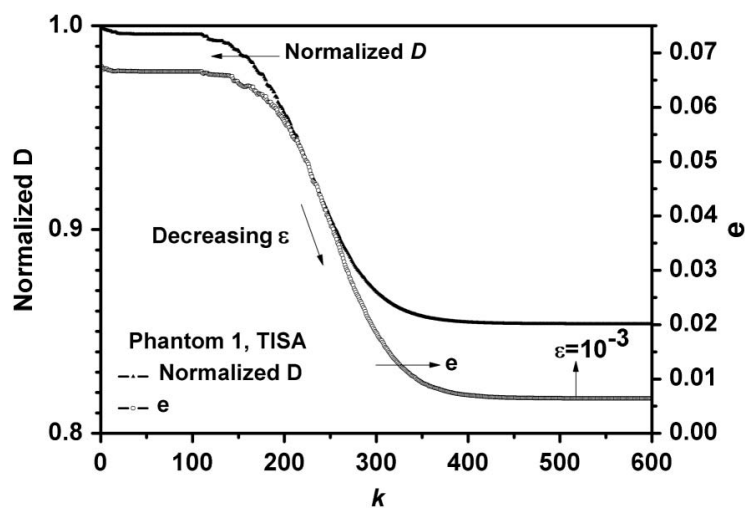

Fig. 5. Evolution of $e$ and normalized $F$ illustrating the monotonic decrease of $e$ in the RHybrid algorithm. 


\section{B. Regularization}

It has been well recognized that a priori information, if available, can be incorporated in the tomographic inversion via regularization to improve the inversion $[12,13,27]$. Here we studied the regularization of the $\overline{\mathrm{ART}}$ and TISA algorithms (the regularized algorithms were code named RART and RTISA, respectively). Two types of regularizations were studied: smoothness and total-variation. The smoothness regularization considers the degree of smoothness of the sought $F$ in the inversion, as detailed in $[12,14]$. The total variation (TV) regularization of the target function $F$ is defined as [13]

$$
\min f=D+\gamma \cdot R_{\mathrm{TV}},
$$

where $f$ is the new master function and $\gamma$ the regularization parameter to adjust the relative importance between the $D$ and $R_{\mathrm{TV}}$ terms. The optimal selection of $\gamma$ is critical for the application of any regularization technique, which controls the relative weights of the a priori information (e.g., smoothness or TV) and the a posteriori knowledge (i.e., the measurements) in the tomographic inversion process [27]. This work found the so-called L-curve method, developed for solving ill-posed linear equations [39], to be effective in determining the optimal $\gamma$ for relatively simple flames.

$$
R_{T V}(F)=\sum_{i_{x}, i_{y}, i_{z}} \sqrt{\left(F_{i_{x}, i_{y}, i_{z}}-F_{i_{x}-1, i_{y}, i_{z}}\right)^{2}+\left(F_{i_{x}, i_{y}, i_{z}}-F_{i_{x}, i_{y}-1, i_{z}}\right)^{2}+\left(F_{i_{x}, i_{y}, i_{z}}-F_{i_{x}, i_{y}, i_{z}-1}\right)^{2}} .
$$

According to Eq. (5), the TV of $F$ represents the summation of the gradient magnitude of $F$ over all voxels. Inclusion of $R_{\mathrm{TV}}$ in the reconstruction has been shown to preserve the smoothness or the edges of the sought $F[10,13]$. In the RART algorithm, the $R_{\mathrm{TV}}$ term was minimized at the end of each ART iteration with respect to $F$, and the updated $F$ was then used as the input for the next ART iteration. In the RTISA algorithm, the $R_{\mathrm{TV}}$ term was simply added to the difference defined in Eq. (2) to form a new master function to be minimized, i.e.,
Before detailing the choice of $\gamma$, Fig. $\underline{6}$ first shows a set of results to illustrate the usefulness of regularization (and also its limitations). We applied the ART algorithms to various phantoms with and without the TV regularization, and two sets of example results are shown in Fig. $\underline{6}$. These results were obtained with phantoms 2 and 4 as shown in Fig. 2 . Eight simulated projections were used in the simulations, with 5\% Gaussian noise artificially added to the projections to simulate measurement uncertainty. The upper panel of Fig. 6 shows the

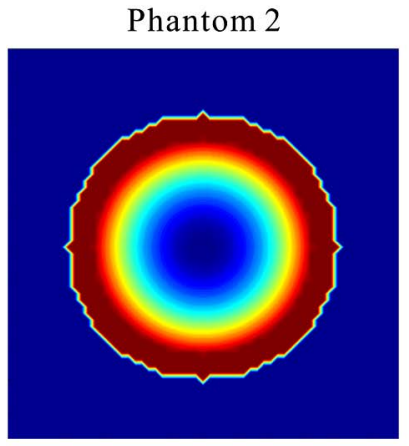

Phantom 4

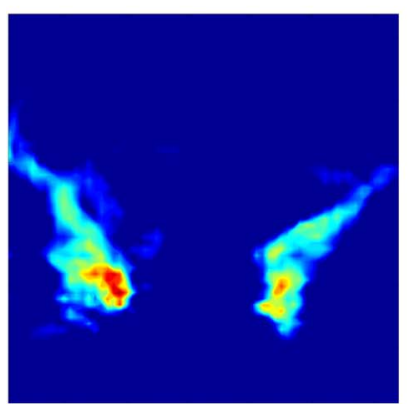

ART, $5.41 \%$ error

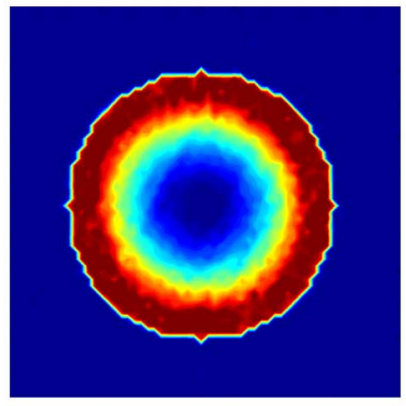

ART, $5.62 \%$ error

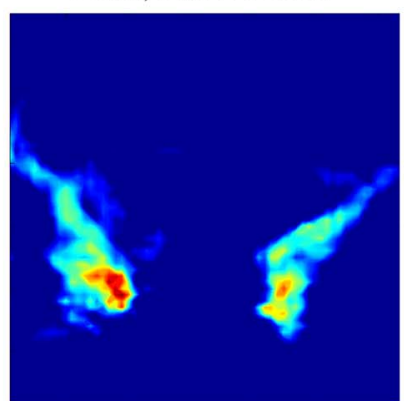

RART, $2.85 \%$ error

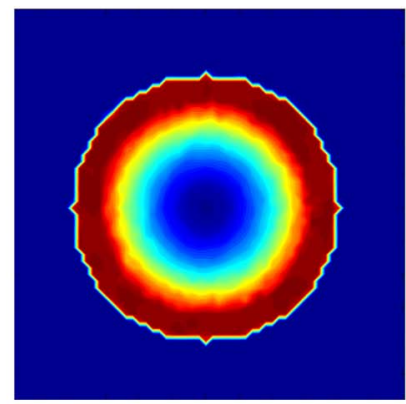

RART, $5.38 \%$ error

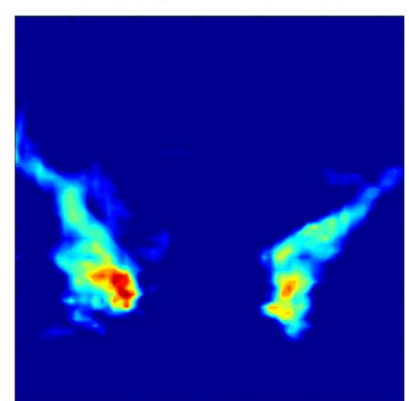

Fig. 6. Application of regularization in the TC technique. Projections from eight random views were used with $5 \%$ Gaussian noise added (these same conditions were used in the results in Figs. $\underline{7}$ and $\underline{8}$ ). 
reconstruction of phantom 2 , which as mentioned earlier, was created to simulate a cone-shaped stable laminar flame generated by a Bunsen burner. Note that even though the phantom is axially symmetric, the tomographic reconstruction did not assume such a priori knowledge. From left to right is the phantom itself, the ART reconstruction, and the RART reconstruction, respectively. As can been seen, the ART reconstruction had artifacts (e.g., the discontinuities and cavities in the reconstruction) and the overall error was $e=5.6 \%$. The application of the TV regularization significantly reduced the overall error to $e=2.8 \%$ and eliminated much of the artifacts seen in the ART reconstruction.

The lower panel of Fig. $\underline{6}$ shows the reconstruction of phantom 4, which was created to simulate a turbulent flame stabilized on a v-gutter. Again from left to right is the phantom itself, the ART reconstruction, and the RART reconstruction, respectively. As can be seen, the ART and RART reconstructions were almost identical to each other. The overall error of the ART reconstruction was $e=5.6 \%$, and that of the RART reconstruction is $e=5.4 \%$.

Several observations can be made from the results shown in Fig. $\underline{6}$, and these observations were valid when we applied regularization to other algorithms (e.g., the TISA algorithm) and other phantoms. First, with a proper choice of $\gamma$ (to be discussed immediately below), the application of regularization reduced $e$ for all algorithms on all phantoms tested. Second, however, the reduction was more pronounced on smooth (or "laminar") phantoms than on irregular (or "turbulent") phantoms, because the TV regularization preserves the smoothness and sharp edges. In the examples shown in Fig. $\underline{6}$, phantom 2 is smooth and has clear edges and therefore the TV regularization is effective, but phantom 4 does not feature any clear edge or smooth distribution, causing the ineffectiveness of the TV regularization as reflected in Fig. 6. It is an important research topic to find a regularization that can work effectively on turbulent
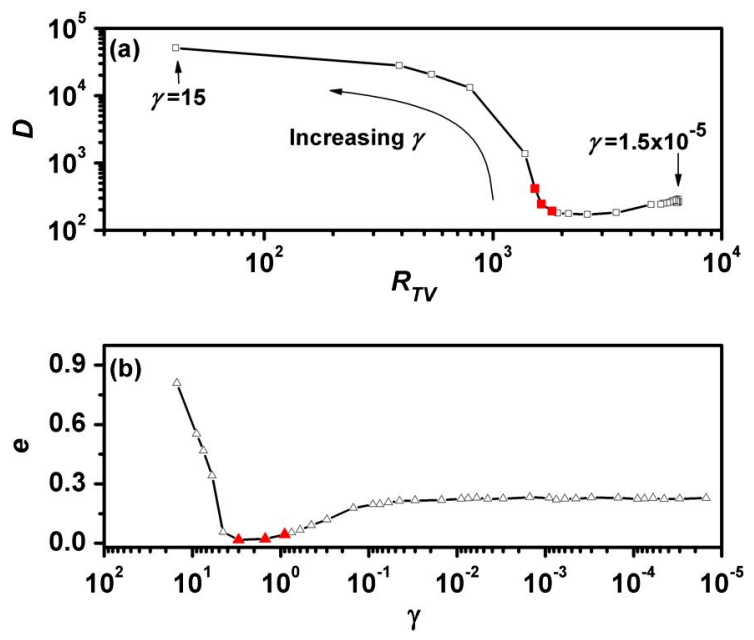

Fig. 7. L curve for phantom 2 (a smooth flame).
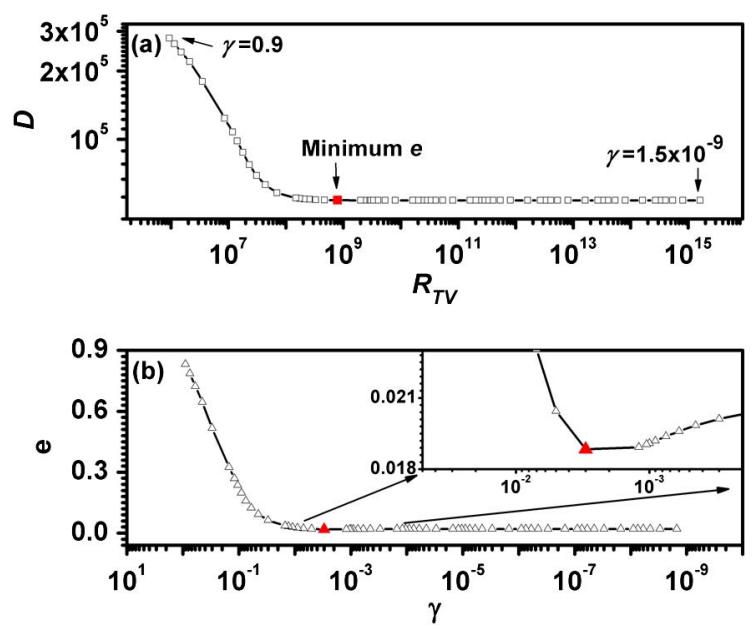

Fig. 8. Application of regularization to phantom 4 (a turbulent flame).

targets, and we are exploring the incorporation of governing equations as regularization in our ongoing work.

Figures 7 and 8 provide more insights into results shown in Fig. 6, and also illustrate the selection of $\gamma$ using the L-curve method. In practice, the sought $F$ is unknown, and therefore $e$ is not available to guide the selection of $\gamma$. The L-curve method recognizes this issue and therefore relies on quantities that can be practically obtained to determine $\gamma$ : the difference between the measured and calculated projections $(D)$ and the regularization term itself (e.g., $R_{\mathrm{TV}}$ ). In the L-curve method, the inversion problem is solved multiple times, each time with a different $\gamma$, and $D$ and the regularization term are recorded each time. Panel (a) of Fig. 7 shows a set of results of $D$ and $R_{\text {TV }}$ recorded when the TV regularization was applied to phantom 2 using the RTISA method. The plot had an approximate $\mathrm{L}$ shape, and the L-curve method used the $\gamma$ corresponding to the corner of the L curve as the optimal values. This work calculated the maximum curvature to determine the corner, and the points near the corner were shown as solid square symbols in Panel (a) of Fig. 7. Panel (b) of Fig. 7 shows the $e$ obtained under the $\bar{\gamma}$ s used (because a known phantom was used here so $e$ can be calculated). The solid triangle symbols correspond to the $\gamma \mathrm{s}$ near the corner of the L curve shown in Panel (a), illustrating that the minimal $e$ indeed occurred at the $\gamma$ s determined by the L-curve method. The L curve exemplified in Panel (a) of Fig. 7 is essentially a trade-off curve. When $\gamma$ is negligibly small (i.e., $1.5 \times 10^{-5}$ ), the inversion was performed only to minimize $D$ without considering the regularization, resulting in small $D$ and large $R_{\text {TV }}$. When $\gamma$ is exceedingly large (i.e., $15)$, the inversion was performed only based on the regularization (i.e., to minimize the TV) without considering the measurements, resulting in large $D$ and small $R_{\mathrm{TV}}$. The success of the L-curve method in the case shown in Fig. 7 lies in the existence of a distinct corner during the transition from small to large $\gamma$, as 
shown in Panel (a). Such a distinct corner represents an optimal balance between $D$ and $R$. Any further increase in $\gamma$ leads to a sharp rise in $D$, and any decrease in $\gamma$ results in negligible change in $D$. Therefore, the $\gamma \mathrm{s}$ near the corner represent a state where the maximum "amount" of regularization can be added in the inversion without affecting the role of the measurements.

In contrast, the data shown in Fig. $\underline{8}$ obtained on a turbulent phantom do not exhibit such a distinct corner. As shown in Panel (a), the transition from small to large $\gamma$ was gradual in this case, resulting in the failure to identify the optimal $\gamma$ and explaining the marginal usefulness of regularization observed in Fig. 6 .

Application of the smoothness regularization to the phantoms showed a similar trend as seen in Figs. 7 and 8. Both the smoothness and TV regularization were effective in improving the inversion on phantoms that are smooth and/or have clear edges, but not effective on turbulent phantoms. The design of an effective regularization technique for turbulent objects is an important research need.

Lastly, the studies describe in Sections A and B above were also performed using experimental data, and the same observations were made as those made with numerical phantoms. The experimental flames were not as accurately known as the numerical phantoms. Therefore, the studies involving experimental data relied on some characteristic features extracted from the flames, and such extraction is best explained in Sections C and D below.

\section{Number of Views and Resolution of Projection Measurements}

The number of views and resolution of the projection measurements are also two important aspects for the
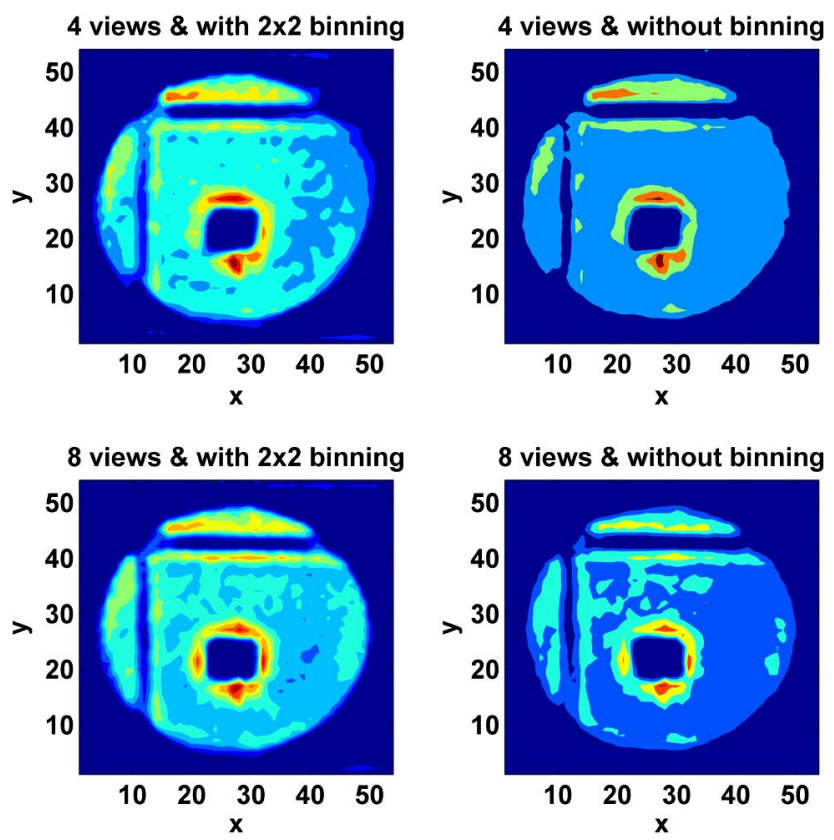

Fig. 9. Layer 1 of the reconstructions from experimentally measured projections. practical implementation of 3D diagnostics. They directly impact the requirement of optical access and cost (both hardware cost and computational cost). Therefore, this section investigates their effects on the quality of the 3D measurements.

Figure 9 shows the reconstruction using experimental data of a flame generated using the setup discussed in Section 2.B. To examine the effects of number of views $(N)$ and resolution of the projections measurements, the reconstruction was performed under four different cases by varying the number of views $(N=4$ and 8$)$ and applying binning to the projections $(2 \times 2$ binning and no binning). Without binning, projections measured by the CCD camera $(1376 \times 1040$ pixels with $6.45 \times 6.45 \mu \mathrm{m}$ pixel size $)$ were directly used in the inversion. The measurement domain was discretized into $64 \times 64 \times 16$ voxels (a total of 65,536 voxels). Under these conditions, the PSD described in Eq. (1) at one view angle was about $8 \mathrm{~GB}$ in size when stored in double precision. The size of the PSD is approximately proportional to the number of pixels in the projection measurements. Therefore, with $2 \times 2$ binning, the size of the PSD reduced to 2 GB per view angle. Such memory requirement and computational cost underline the importance to carefully design the number of views and resolution of the projections in practice. Figure $\underline{9}$ shows that (1) eight views resulted in an overall more accurate reconstruction than four views and (2) with a fixed number of views, reducing the resolution of the projections via binning does not significantly deteriorate the overall quality of the reconstruction. One explanation for the second observation is that while binning reduced the resolution and thusly reduced the number of measurements available as inputs for the inversion, it also reduced the uncertainty in the measurements at the same time.

Figure 10 shows a quantitative analysis of the results in Fig. 9 by focusing on the vertical column blocked in the flame. As mentioned in Section 2.B, the blocked column had a width of $1.25 \mathrm{~mm}$, representing the smallest spatial feature created in the

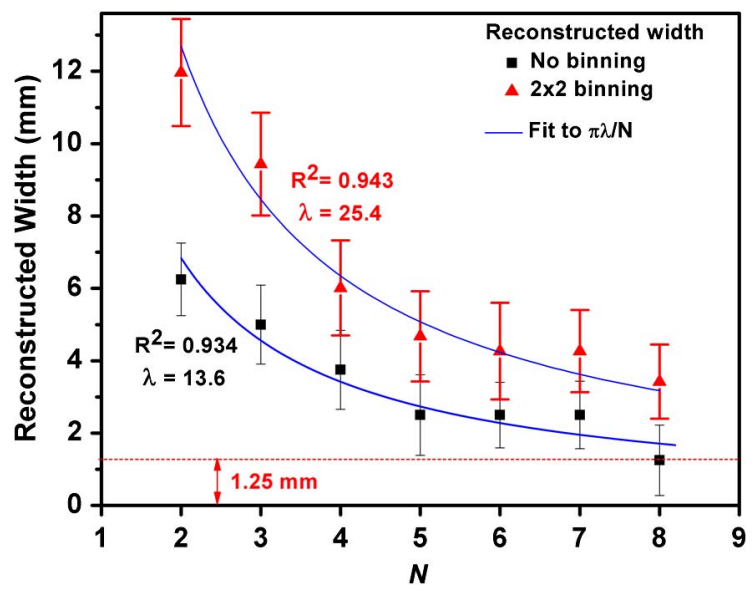

Fig. 10. Reconstruction of experimental data with and without binning the measured projections. 
experiments. Based on the reconstructions shown in Fig. 9, we extracted the width of the column by calculating the gradient of the reconstruction and locating the sharpest $\mathrm{CH}^{*}$ concentration change. Figure 10 shows the reconstructed width of the column under the conditions used in Fig. 9 . Note that the reconstructed width may vary along the column (i.e., at difference $y$ locations). Therefore, multiple values were obtained from each layer and Fig. 10 shows the median (the solid symbols) and standard deviation (the error bars) of these values.

Several observations can be made based on the results shown in Fig. 10. First, the spatial resolution of the reconstruction, quantified by the width of the blocked column, improved with increasing number of views used. Figure 10 also shows the fit of the results to the Fourier slice theorem $[40,41]$, which predicts the spatial resolution of the reconstruction to be $\pi \lambda / N$, where $\lambda$ is a characteristic spatial scale. As seen, the data were accurately captured by the theorem. Second, different $\lambda$ was determined when binning was applied to the measured projections, leading to a different resolving power of the tomographic inversion. Therefore, even though the results shown in Fig. 9 suggest that binning did not cause significant degradation to the overall reconstruction quality, the quantitative analysis shown in Fig. 10 show that binning does affect the resolving power of the tomographic inversion. Third, when projection data from eight views were used without binning, the tomographic inversion was able to resolve the minimum feature in the flame $(1.25 \mathrm{~mm})$, demonstrating the spatial resolution of the 3D diagnostic technique.

Figures 11 and 12 show numerical results performed using phantoms to simulate the experiments described above. As can be seen, the same
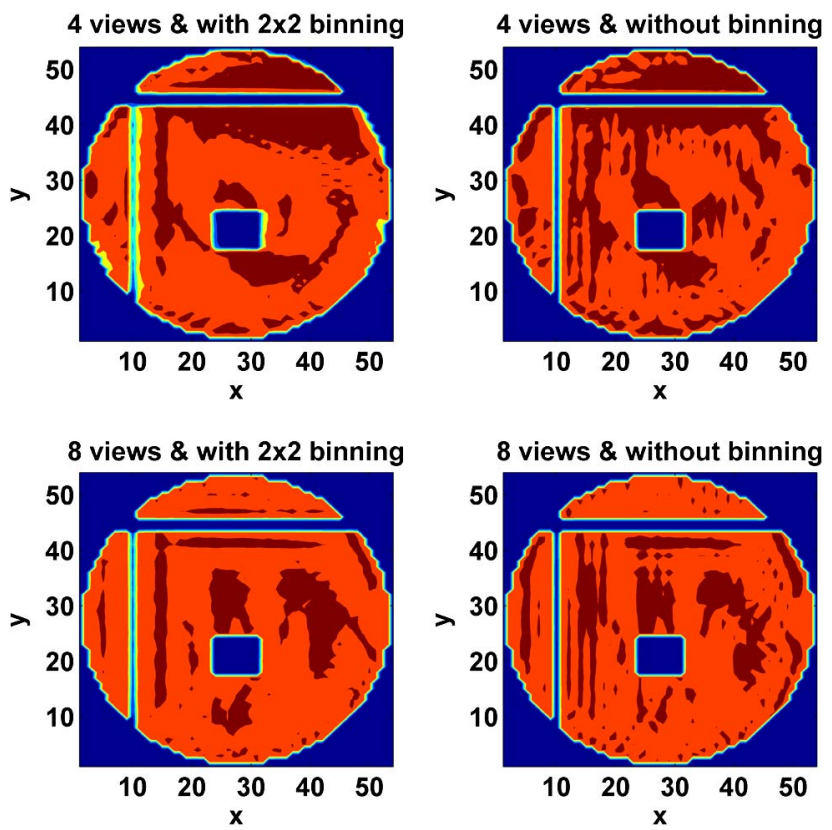

Fig. 11. Layer 1 of the reconstructions from simulated projections.

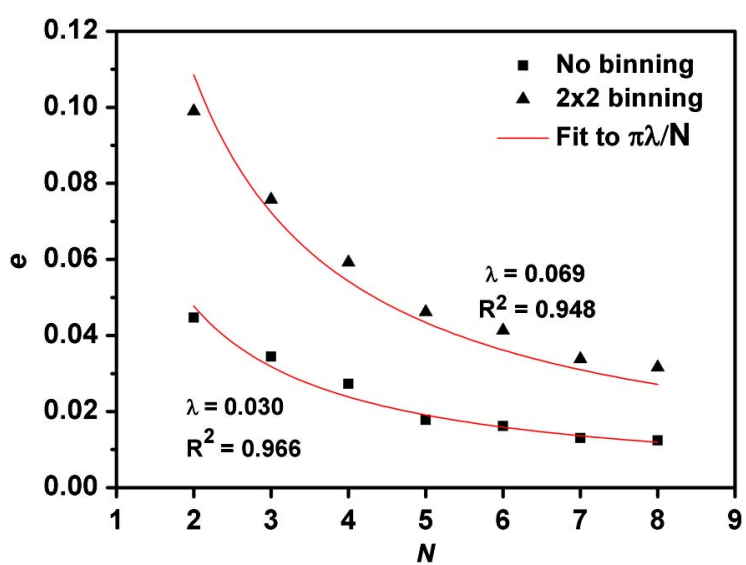

Fig. 12. Reconstruction of experimental data with and without binning the simulated projections.

observations can be made from these numerical results as those made from the experimental results. Finally, we make two notes before leaving these discussions. First, in the numerical simulations, both the width of the blocked column and $e$ can be calculated due to the precisely known phantoms. The reconstructed width and its fit showed the same trend as those seen in Fig. 10 from the experimental data. Therefore, here Fig. 12 shows $e$ from the numerical simulations rather than the width of the blocked column. The results in Fig. 12 show that $e$ can be fitted accurately by the Fourier slice theorem too, which could be useful for quantifying the inversion accuracy of targets with no distinct spatial features. Second, the Fourier slice theorem was developed for the ART algorithm, and results obtained in this work suggested that it also applies to the TISA algorithm.

Lastly, in practice, the number of views is an important parameter in the design of tomographic combustion diagnostics. These observations made here illustrate the practical factors that should be considered in determining the optical number of views. These factors include fundamental considerations such as the desired spatial resolution and pragmatic factors such as the computational cost and optical access. The results shown here should provide valuable guidance to the holistic consideration of these factors.

\section{Summary and Discussion}

This paper investigated four practical aspects for implementing 3D tomographic inversion under the context of volumetric flame imaging. These aspects include: (1) the termination criteria of the inversion algorithm; (2) the effects of regularization and the determination of the optimal regularization factor; (3) the effects of number of views, and (4) the impact of the resolution of the projection measurements. Both numerical simulations and controlled experiments were performed to study them. The results obtained have shown the difficulties of designing an effective termination criterion, and suggested that 
the new TISA algorithm can be terminated effectively on all the cases tested. Regularization has been demonstrated to significantly enhance the accuracy of inverting smooth flames and/or flames with clear edges. An L-curve method was found to be able to determine the optimal regularization parameter. Increasing the number of views and the resolution of the projections has been shown to improve both the accuracy and the resolving power, which agreed with theoretical predictions. The results obtained have illustrated the effects of these practical aspects on the accuracy and spatial resolution of 3D diagnostics based on tomography inversion. Furthermore, these aspects, for instance the number of views and the resolution of the projection measurements, are all related to the complexity and implementing cost (both hardware cost and computational cost). Therefore, we expect the results obtained in this work to facilitate the practical implementation of $3 \mathrm{D}$ combustion diagnostics. For example, in our ongoing work, we are designing temporally-resolved 3D diagnostics for practical combustors, which pose several challenges including restricted optical access, large measurement volume, and large data volume. The results discussed in this work provide key information to many aspects of the design, which aims at obtaining an optimal balance of spatial resolution, temporal resolution, hardware cost, and computational cost.

\section{References}

1. G. J. Nathan, P. A. M. Kalt, Z. T. Alwahabi, B. B. Dally, P. R. Medwell, and Q. N. Chan, "Recent advances in the measurement of strongly radiating, turbulent reacting flows," Prog. Energy Combust. Sci. 38, 41-61 (2012).

2. R. S. Barlow, "Laser diagnostics and their interplay with computations to understand turbulent combustion," Proc. Combust. Inst. 31, 49-75 (2007).

3. L. Ma, "High speed imaging in reactive flows using hyperspectral tomography and photodissociation spectroscopy," in Laser Applications to Chemical, Security and Environmental Analysis, OSA Technical Digest (Optical Society of America, 2010), paper LWA3.

4. L. Ma, X. Li, S. Roy, A. Caswell, J. R. Gord, D. Plemmons, X. An, and S. T. Sanders, "Demonstration of high speed imaging in practical propulsion systems using hyperspectral tomography," in Laser Applications to Chemical, Security and Environmental Analysis, OSA Technical Digest (Optical Society of America, 2012), paper LM1B.5.

5. R. Wellander, M. Richter, and M. Alden, "Time resolved, 3D imaging (4D) of two phase flow at a repetition rate of $1 \mathrm{kHz}$," Opt. Express 19, 21508-21514 (2011).

6. J. Hult, A. Omrane, J. Nygren, C. F. Kaminski, B. Axelsson, R. Collin, P. E. Bengtsson, and M. Alden, "Quantitative three-dimensional imaging of soot volume fraction in turbulent non-premixed flames," Exp. Fluids 33, 265-269 (2002).

7. D. P. Correia, P. Ferrao, and A. Caldeira-Pires, "Advanced 3D emission tomography flame temperature sensor," Combust. Sci. Technol. 163, 1-24 (2001).

8. R. Snyder and L. Hesselink, "Measurement of mixing fluidflows with optical tomography," Opt. Lett. 13, 87-89 (1988).

9. J. Floyd, P. Geipel, and A. M. Kempf, "Computed tomography of chemiluminescence (CTC): instantaneous 3D measurements and phantom studies of a turbulent opposed jet flame," Combust. Flame 158, 376-391 (2011).

10. X. Li, W. Cai, F. Li, and L. Ma, "Numerical and experimental validation of a three-dimensional combustion diagnostic based on tomographic chemiluminescence," Opt. Express 21, 7050-7064 (2013).

11. C. T. Herman, Image reconstruction from projections-the fundamentals of computerized tomography (Academic, 1980).

12. L. Ma and W. Cai, "Determination of the optimal regularization parameters in hyperspectral tomography," Appl. Opt. 47, 4186 (2008)

13. E. Y. Sidky, C. M. Kao, and X. H. Pan, "Accurate image reconstruction from few-views and limited-angle data in divergent-beam CT," J. X-Ray Sci. Technol. 14, 119-139 (2006).

14. W. Cai, D. J. Ewing, and L. Ma, "Application of simulated annealing for multispectral tomography," Comput. Phys. Commun. 179, 250 (2008).

15. X. An, T. Kraetschmer, K. Takami, S. T. Sanders, L. Ma W. Cai, X. Li, S. Roy, and J. R. Gord, "Validation of temperature imaging by $\mathrm{H} 2 \mathrm{O}$ absorption spectroscopy using hyperspectral tomography in controlled experiments," Appl. Opt. 50, A29-A37 (2011).

16. L. Ma, W. Cai, A. W. Caswell, T. Kraetschmer, S. T. Sanders, S. Roy, and J. R. Gord, "Tomographic imaging of temperature and chemical species based on hyperspectral absorption spectroscopy," Opt. Express 17, 8602-8613 (2009).

17. L. Ma, X. Li, S. T. Sanders, A. W. Caswell, S. Roy, D. H. Plemmons, and J. R. Gord, "50-kHz-rate 2D imaging of temperature and $\mathrm{H} 2 \mathrm{O}$ concentration at the exhaust plane of a J85 engine using hyperspectral tomography," Opt. Express 21, 1152-1162 (2013).

18. Q. Huang, F. Wang, J. Yan, and Y. Chi, "Simultaneous estimation of the 3-D soot temperature and volume fraction distributions in asymmetric flames using high-speed stereoscopic images," Appl. Opt. 51, 2968-2978 (2012).

19. J. Floyd and A. M. Kempf, "Computed tomography of chemiluminescence (CTC): high resolution and instantaneous 3-D measurements of a matrix burner," Proc. Combust. Inst. 33, $751-758$ (2011).

20. M. M. Hossain, G. Lu, and Y. Yan, "Optical fiber imaging based tomographic reconstruction of burner flames," IEEE Trans. Instrum. Meas. 61, 1417-1425 (2012).

21. W. Cai, A. J. Wickersham, and L. Ma, "Three-dimensional combustion diagnostics based on computed tomography of chemiluminescence," presented at the 51st AIAA Aerospace Sciences Meeting Including the New Horizons Forum and Aerospace Exposition, Dallas Region, Texas, 7-10 January 2013.

22. L. Ma, L. Kranendonk, W. Cai, Y. Zhao, and J. Baba, "Application of simulated annealing for simultaneous retrieval of particle size distribution and refractive index," J. Aerosol Sci. 40, 588-596 (2009).

23. W. Cai, L. Kranendonk, T. Lee, and L. Ma, "Characterization of composite nanoparticles using an improved light scattering program for coated spheres," Comput. Phys. Commun. 181, 978-984 (2010).

24. Y. Zhao, X. Li, and L. Ma, "Multidimensional Monte Carlo model for two-photon laser-induced fluorescence and amplified spontaneous emission," Comput. Phys. Commun. 183, 1588-1595 (2012).

25. R. Crowther, D. DeRosier, and A. Klug, "The reconstruction of a three-dimensional structure from projections and its application to electron microscopy," Proc. R. Soc. London 317, 319-340 (1970)

26. H. M. Hudson and R. S. Larkin, "Accelerated image reconstruction using ordered subsets of projection data," IEEE Trans. Med. Imaging 13, 601-609 (1994).

27. W. H. Press, S. A. Teukolsky, W. T. Vetterling, and B. P. Flannery, Numerical Recipes in FORTRAN: The Art of Scientific Computing (Cambridge, 1992).

28. W. Cai and L. Ma, "Comparison of approaches based on optimization and algebraic iteration for binary tomography," Comp. Phys. Commun. 181, 1974-1981 (2010).

29. X. Li and L. Ma, "Minimizing binary functions with simulated annealing algorithm with applications to binary tomography," Comput. Phys. Commun. 183, 309-315 (2012).

30. Y. Ishino and N. Ohiwa, "Three-dimensional computerized tomographic reconstruction of instantaneous distribution of 
chemiluminescence of a turbulent premixed flame," JSME Int. J. 48, 34-40 (2005).

31. N. Anikin, R. Suntz, and H. Bockhorn, "Tomographic reconstruction of the $\mathrm{OH}^{*}$-chemiluminescence distribution in premixed and diffusion flames," Appl. Phys. 100, 675-694 (2010).

32. J. Kitzhofer, T. Nonn, and C. Bruecker, "Generation and visualization of volumetric PIV data fields," Exp. Fluids 51, 14711492 (2011).

33. W. Cai and L. Ma, "Applications of critical temperature in minimizing functions of continuous variables with simulated annealing algorithm," Comput. Phys. Commun. 181, 11-16 (2010).

34. W. Cai, D. J. Ewing, and L. Ma, "Investigation of temperature parallel simulated annealing for optimizing continuous functions with application to hyperspectral tomography," Appl. Math. Comput. 217, 5754-5767 (2011).

35. A. Seppanen, A. Voutilainen, and J. P. Kaipio, "State estimation in process tomography-reconstruction of velocity fields using EIT," Inverse Probl. 25, 085009 (2009).
36. W. Cai and L. Ma, "Hyperspectral tomography based on proper orthogonal decomposition as motivated by imaging diagnostics of unsteady reactive flows," Appl. Opt. 49, 601-610 (2010).

37. D. Verhoeven, "Limited-data computed-tomography algorithms for the physical sciences," Appl. Opt. 32, 3736-3754 (1993).

38. S. Kirkpatrick, C. D. Gelatt, Jr., and M. P. Vecchi, "Optimization by simulated annealing," Science 220, 671-680 (1983).

39. P. C. Hansen, "Analysis of discrete ill-posed problems by means of the L-Curve," Siam Rev. 34, 561-580 (1992).

40. G. Frieder and G. T. Herman, "Resolution in reconstructing objects from electron micrographs," J. Theor. Biol. 33, 189211 (1971).

41. G. T. Herman and S. Rowland, "Resolution in algebraic reconstruction technique: an experimental investigation of the resolving power of an algebraic picture reconstruction technique," J. Theor. Biol. 33, 213-223 (1971). 